\title{
Avaliação pré-operatória da força muscular respiratória, da função pulmonar e da capacidade funcional de pacientes submetidos a ressecção pulmonar
}

\author{
Preoperative evaluation of respiratory muscle strength, lung function and \\ functional capacity of patients undergoing lung resection
}

Letícia Baltieri', Ana Isabela Morsch Passos ${ }^{1}$, Fernanda Diório Masi Galhardoํ․ Lígia dos Santos Roceto', Ivan Felizardo Contrera Toro²

'Departamento de Fisioterapia da Universidade Estadual de Campinas (UNICAMP) - Campinas (SP), Brasil.

${ }^{2}$ Departamento de Medicina da UNICAMP - Campinas (SP), Brasil.

DOI: http://dx.doi.org/10.7322/abcshs.v40i1.699

\begin{abstract}
RESUMO
Introdução: As neoplasias pulmonares podem causar deterioração na função pulmonar e capacidade funcional, prejudicando a evolução do paciente após cirurgia de ressecção. Objetivo: Avaliar a força muscular respiratória, a função pulmonar e a capacidade funcional de pacientes no pré-operatório de ressecção pulmonar por neoplasia, comparar aos valores de normalidade e por estadiamento. Métodos: Foram incluídos pacientes na faixa etária de 30 a 80 anos com diagnóstico de neoplasia de pulmão que aguardavam cirurgia de ressecção pulmonar. A avaliação pré-operatória incluiu medida de força muscular respiratória por manovacuometria, função pulmonar por espirometria e capacidade funcional pelo teste de caminhada dos seis minutos (TC6). Os valores obtidos foram comparados aos valores preditos pela literatura e comparados os estadiamentos inicial e avançado. Resultados: Foram avaliados 59 pacientes com idade média de 58,5 $\pm 9,46$ anos. Houve diferença estatisticamente significante entre obtido e predito, respectivamente, para os valores de pressão expiratória máxima (PEmáx): 91 e 111,8 cmH $\mathrm{cm}_{2} \mathrm{O}$; capacidade vital forçada (CVF): 3,14 e 4,08 L; volume expiratório forçado no primeiro segundo (VEF $F_{1}$ : 2,42 e 3,12 L; razão VEF/CVF: 75 e 101\%; e capacidade funcional: 453,9 e 562,9 m, sendo que os pacientes apresentaram valores inferiores aos preditos pela literatura, exceto a CVF. Não houve diferença para a pressão inspiratória máxima (PImáx): 110 e $102 \mathrm{cmH}_{2} \mathrm{O}$. Entre os grupos de estadiamento houve diferença apenas para a CVF (inicial: 2,89; avançado: 3,41 L). Conclusão: Os pacientes com diagnóstico de neoplasia pulmonar em pré-operatório de ressecção possuem prejuízos na força muscular expiratória, na função pulmonar e na capacidade funcional.
\end{abstract}

Palavras-chave: neoplasias pulmonares; espirometria; testes de função respiratória; fisioterapia.

\begin{abstract}
Introduction: Lung cancer can cause deterioration in lung function and functional capacity, impairing patient outcome after resection. Objective: To evaluate the respiratory muscle strength, lung function and functional capacity of patients in preoperative of lung resection for cancer, compared to normal values and staging. Methods: Patients of both genders were included aged $30-80$ years with a diagnosis of lung cancer awaiting pulmonary resection surgery. Preoperative evaluation included measurement of respiratory muscle strength by manometer, lung function by spirometry and functional capacity through the sixminute walk test (6MWT). The values obtained were compared with predicted values obtained from equations proposed in the literature and compared the staging initial and advanced. Results: 59 patients with a mean age of $58.5 \pm 9.46$ years were evaluated. There was a statistically significant difference between the obtained and the predicted values for maximum expiratory pressure (MEP): respectively 91 and $111.8 \mathrm{cmH}_{2} \mathrm{O}$, forced vital capacity (FVC): 3.14 and $4.08 \mathrm{~L}$; forced expiratory volume in the first second $\left(\mathrm{FEV}_{1}\right): 2.42$ and $3.12 \mathrm{~L} ; \mathrm{FEV}_{1} / \mathrm{FVC}$ : 75 and 101\%; and functional capacity: 453.9 and $562.9 \mathrm{~m}$, in which the patients had lower values than those predicted by the literature, except FVC. There was no difference for inspiratory muscle strength (MIP: 110 and $102 \mathrm{cmH}_{2} \mathrm{O}$ ). Among the groups of patients only showed a difference of FVC (initial: 2.89; advanced: $3.41 \mathrm{~L}$ ). Conclusion: Patients diagnosed with lung cancer in preoperative of resection, have impairments in expiratory muscle strength, lung function and functional capacity.
\end{abstract}

Keywords: lung neoplasms; spirometry; respiratory function tests; physical therapy specialty. 


\section{INTRODUÇÃO}

Atualmente, as neoplasias constituem um problema de saúde pública, sendo o câncer de pulmão o mais comum dos tumores malignos. Estima-se que haja 27.330 casos novos em 2014, com um número estimado de mortes de 22.424 casos $^{1}$. Hoje, a cirurgia de ressecção pulmonar é uma alternativa no tratamento do paciente, apresentando uma mortalidade em 3 anos de $15,4 \%^{2}$.

Aproximadamente $90 \%$ de todos os carcinomas broncogênicos estão ligados ao tabagismo ${ }^{1}$. Em geral, os tabagistas possuem uma incidência de câncer no pulmão 10 a 25 vezes maior em comparação aos não tabagistas. $\mathrm{O}$ carcinoma broncogênico, assim como outras lesões malignas, é resultante de estímulos que lesam o material genético, e comprovadamente o tabaco provoca alterações morfológicas na célula epitelial da via aérea e crescimento anormal, prejudicando as funções do respectivo órgão ${ }^{3}$.

Alguns fatores influenciam a sobrevida dos pacientes com diagnóstico de carcinoma não pequenas células de pulmão, dentre eles a capacidade funcional ${ }^{4}$. Desse modo, existe evidência de que a ressecção pulmonar gera deterioração da função pulmonar e da capacidade ao exercício ${ }^{5-8}$. Nesse sentido, a neoplasia pulmonar por si só pode causar prejuízos na função pulmonar e na capacidade funcional ${ }^{4}$; caso esse prejuízo já exista no período pré-operatório, o paciente deve ser foco de atenção do fisioterapeuta para o tratamento direcionado e a redução dos riscos pós-cirúrgicos.

Em pacientes submetidos a ressecção pulmonar um volume expiratório forçado no primeiro segundo $\left(\mathrm{VEF}_{1}\right)$ abaixo de $2 \mathrm{~L} / \mathrm{s},{ }^{8}$ ou qualquer anormalidade na prova de função pulmonar', demonstrou aumentar a frequência de complicações pós-operatórias.

Portanto, é essencial a triagem pré-operatória desses pacientes, além da análise e classificação do estadiamento do tumor, pois ao avaliar a extensão da doença é possível determinar o planejamento terapêutico e o prognóstico do caso ${ }^{10}$.

A hipótese deste estudo foi a de que o indivíduo portador de neoplasia pulmonar possui comprometimento da função pulmonar, da força muscular respiratória e da capacidade funcional antes da exposição cirúrgica, fato este que comprometerá a evolução pós-operatória, quando indicada. Além disso, acredita-se que pacientes em estadiamento mais avançado estejam com tais funções mais debilitadas em comparação aos pacientes em estadiamento inicial. Caso seja comprovado que há prejuízo na função pulmonar, na força muscular respiratória e na capacidade funcional do paciente, e haja diferença entre os estadiamentos, é justificada a necessidade de um programa de reabilitação pulmonar pré-operatório e preparo do paciente para a cirurgia eletiva.

Sendo assim, o objetivo do estudo foi avaliar a força muscular respiratória, a função pulmonar e a capacidade funcional de pacientes no pré-operatório de ressecção pulmonar por neoplasia, além de comparar aos valores previstos na literatura e entre grupos de estadiamento.

\section{MÉTODOS}

Trata-se de estudo prospectivo realizado no Hospital de Clínicas da Universidade Estadual de Campinas (HC-UNICAMP). O trabalho foi aprovado pelo Comitê de Ética em Pesquisa da instituição, sob o protocolo n ${ }^{\circ} 388 / 2007$, e todos os voluntários assinaram um termo de consentimento.

Os pacientes foram triados semanalmente nas enfermarias do hospital no momento da internação para agendamento cirúrgico.

Foram incluídos pacientes de ambos os gêneros, na faixa etária de 30 a 80 anos, com diagnóstico médico de neoplasia de pulmão que aguardavam as seguintes cirurgias de ressecção pulmonar: lobectomia, bilobectomia ou pneumectomia por toracotomia posterolateral.

A avaliação pré-operatória foi realizada por fisioterapeutas treinados e incluiu anamnese com coleta de dados antropométricos, hábitos de vida e consulta do prontuário, para coleta do estadiamento do câncer, além de exame físico, medida de força muscular respiratória, função pulmonar e capacidade funcional. As medidas foram realizadas sempre pelos mesmos profissionais.

\section{Estadiamento do câncer}

A análise do estadiamento do câncer foi realizada pelo médico responsável pelo caso do paciente e anotada em prontuário. A análise foi baseada na Classificação TNM elaborada pela União Internacional contra o Câncer (UICC), traduzida pelo Instituto Nacional de Câncer (INCA) ${ }^{10}$. Os pesquisadores obtiveram os dados dos prontuários dos pacientes, porém alguns não possuíam a informação ou não constavam no prontuário.

\section{Força muscular respiratória}

A força muscular respiratória foi realizada por meio da medida das pressões respiratórias máximas (pressão inspiratória máxima - PImáx - e pressão expiratória máxima - PEmáx), utilizando um manovacuômetro analógico (Comercial Médica São Paulo, Brasil), com intervalo operacional de $\pm 120 \mathrm{cmH}_{2} \mathrm{O}$.

Utilizando um clipe nasal, a PImáx foi medida a partir de uma expiração máxima (próximo ao volume residual) e da solicitação de uma inspiração máxima e profunda no bocal do equipamento. A PEmáx foi medida a partir de uma inspiração máxima (próximo à capacidade pulmonar total) e da solicitação de uma expiração máxima e profunda no bocal do equipamento. Cada esforço foi sustentado por, no mínimo, 2 segundos, com 45 segundos de intervalo entre cada manobra. Para minimizar o efeito aprendizagem, foram realizadas 3 medidas, sendo que a diferença entre elas deveria ser de até $10 \%$. Foi considerado para análise o maior valor obtido.

Os valores obtidos foram comparados às equações de normalidade sugeridas por Neder et al. ${ }^{11}$.

\section{Função pulmonar}

A prova de função pulmonar foi obtida por meio da espirometria realizada em laboratório de função pulmonar do 
hospital e consultada pelos pesquisadores no prontuário médico do paciente. As manobras espirométricas foram padronizadas de acordo com normas da American Thoracic Society (ATS) e da European Respiratory Society (ERS) ${ }^{12}$.

Para calcular os valores preditos, foram utilizadas as equações propostas por Pereira et al..$^{13}$ para a população brasileira.

\section{Capacidade funcional}

A capacidade funcional foi obtida a partir da distância percorrida no teste de caminhada dos seis minutos (TC6) realizado de acordo com as recomendações da $\mathrm{ATS}^{14}$.

O teste foi realizado em corredor plano de $30 \mathrm{~m}$ e os participantes receberam instruções para caminhar durante 6 minutos do início ao final do corredor o mais rápido que conseguissem ${ }^{14}$. Os valores alcançados foram comparados às equações de referência sugeridas por Britto et al. ${ }^{15}$ para a população brasileira.

\section{Análise estatística}

Foi utilizado o programa estatístico SPSS, versão 15.0. Foi realizada a análise descritiva das variáveis numéricas por meio de medida de tendência central e dispersão e de frequência das variáveis categóricas. Foi realizado o teste de normalidade de Shapiro-Wilk para as variáveis de desfecho; não havendo normalidade dos dados de força muscular respiratória e função pulmonar (capacidade vital forçada - CVF - e razão entre volume expiratório forçado no primeiro segundo e capacidade vital forçada $-\mathrm{VEF}_{1} / \mathrm{CVF}$ ), foi realizado o teste de Wilcoxon. Foi realizado o teste $\mathrm{T}$ para amostras relacionadas aos dados da distância percorrida no TC6 e à função pulmonar $\left(\mathrm{VEF}_{1}\right)$, já que houve distribuição normal dos dados. Para a análise dos pacientes divididos em grupos de estadiamento, foram utilizados o teste $\mathrm{T}$, para amostras independentes para as variáveis que apresentaram normalidade (PEmáx, CVF, $V_{1} F_{1}$ e TC6), e o teste de Mann-Whitney, para as variáveis que não apresentaram normalidade (PImáx e VEF $/ C V F$ ). Foi considerado diferença estatisticamente significante um valor de $\mathrm{p}<0,05$.

\section{RESULTADOS}

Foram avaliados 59 pacientes de ambos os gêneros. As Tabelas 1 e 2 resumem as características demográficas e antropométricas da amostra estudada, bem como os dados relacionados a tabagismo e tipo de cirurgia proposta. A Tabela 3 apresenta os valores do estadiamento do câncer de 30 pacientes, sendo a maioria classificada como estádio IIIA.

Tabela 1: Idade, peso, altura e índice de massa corporal dos pacientes estudados $(n=59)$

\begin{tabular}{|l|c|c|}
\hline & Média \pm DP & Variação (mín.-máx.) \\
\hline Idade (anos) & $58,5 \pm 9,46$ & $32-80$ \\
\hline Peso $(\mathrm{kg})$ & $71,4 \pm 14,26$ & $42-105$ \\
\hline Altura $(\mathrm{cm})$ & $166,6 \pm 8,55$ & $142-187$ \\
\hline IMC $\left(\mathrm{kg} / \mathrm{m}^{2}\right)$ & $25,5 \pm 4,43$ & $16,4-39,4$ \\
\hline
\end{tabular}

DP: desvio padrão; IMC: índice de massa corporal
A Tabela 4 apresenta os valores obtidos da força muscular respiratória, da função pulmonar e da capacidade funcional comparados aos valores de normalidade da literatura para os 59 pacientes. Foi observada redução da força muscular expiratória, da função pulmonar e da capacidade funcional.

Os pacientes que possuíam o estadiamento descrito em prontuário foram analisados e divididos nos grupos de estadiamento (Tabela 5), sendo considerados em estadiamento inicial os pacientes com classificação I e II e em estadiamento avançado os com classificação III e IV, sendo possível observar diferença entre os grupos apenas para a CVF, em que os pacientes com estadiamento inicial apresentaram valores menores. Não houve diferença para as demais variáveis.

Tabela 2: Gênero, tabagismo e tipo cirúrgico proposto $(n=59)$

\begin{tabular}{|l|l|c|}
\hline \multirow{2}{*}{ Gênero } & Feminino & $\mathbf{n}(\%)$ \\
\cline { 2 - 3 } & Masculino & $22(22,92)$ \\
\hline \multirow{3}{*}{ Tabagismo } & Não tabagista & $37(77,08)$ \\
\hline \multirow{3}{*}{ Tipo de cirurgia } & Tabagista & $10(7,25)$ \\
\hline & Ex-tabagista & $23(33,3)$ \\
\hline & Tabagista passivo & $22(47,83)$ \\
\hline & Lobectomia & $4(11,59)$ \\
\hline & Bilobectomia & $45(53,57)$ \\
\hline & Pneumectomia & $3(7,14)$ \\
\hline
\end{tabular}

Tabela 3: Dados do estadiamento do câncer baseado na Classificação TNM $(n=30)$

\begin{tabular}{|c|c|c|}
\hline \multicolumn{2}{|l|}{ Classificação TNM } & n (\%) \\
\hline \multirow{7}{*}{ Tumor } & TX & $1(3,3)$ \\
\hline & TO & 0 \\
\hline & Tis & 0 \\
\hline & $\mathrm{T} 1$ & $4(13,3)$ \\
\hline & T2 & $13(43,3)$ \\
\hline & T3 & $11(36,6)$ \\
\hline & T4 & $1(3,3)$ \\
\hline \multirow{5}{*}{ Linfonodos regionais } & NX & $1(3,3)$ \\
\hline & NO & $13(43,3)$ \\
\hline & N1 & $8(26,6)$ \\
\hline & N2 & $8(26,6)$ \\
\hline & N3 & 0 \\
\hline \multirow{3}{*}{ Metástase à distância } & $\mathrm{MX}$ & $1(3,3)$ \\
\hline & MO & $23(76,6)$ \\
\hline & M1 & $6(20)$ \\
\hline \multirow{9}{*}{ Estadiamento } & Carcinoma oculto & 0 \\
\hline & 0 & 0 \\
\hline & IA & $3(10)$ \\
\hline & IB & $6(20)$ \\
\hline & IIA & $1(3,3)$ \\
\hline & IIB & $6(20)$ \\
\hline & IIIA & $8(26,6)$ \\
\hline & IIIB & 0 \\
\hline & IV & $6(20)$ \\
\hline
\end{tabular}


Tabela 4: Força muscular respiratória, função pulmonar e capacidade funcional dos pacientes e o predito na literatura $(n=59)$

\begin{tabular}{|c|c|c|}
\hline & \multicolumn{2}{|c|}{ Média $+\mathrm{DP}$ ou mediana $\left(1^{\circ}\right.$ e $3^{\circ}$ quartis $)$} \\
\hline & Obtido & Predito \\
\hline \multicolumn{3}{|l|}{ Força muscular respiratória } \\
\hline PImáx $\left(\mathrm{cmH}_{2} \mathrm{O}\right)$ & $110(60 ; 120)$ & $102(84,9 ; 108,9)$ \\
\hline PEmáx $\left(\mathrm{cmH}_{2} \mathrm{O}\right)$ & $91(75,5 ; 120)$ & $111,8(83,8 ; 118,32)^{*}$ \\
\hline \multicolumn{3}{|l|}{ Função pulmonar } \\
\hline CVF (L) & $3,14(2,69 ; 3,65)$ & $4,08(3,28 ; 4,47)^{\star}$ \\
\hline $\operatorname{VEF}_{1}(\mathrm{~L})$ & $2,42 \pm 0,62$ & $3,12 \pm 0,59^{\star}$ \\
\hline $\mathrm{VEF}_{1} / \mathrm{CVF}(\%)$ & $75,8(71,1 ; 82,9)$ & $101,1(99,1 ; 103,2)^{*}$ \\
\hline CVF (\% do predito) & \multicolumn{2}{|c|}{$80(70,5 ; 95)$} \\
\hline $\operatorname{VEF}_{1}(\%$ do predito) & \multicolumn{2}{|c|}{$78,7 \pm 18,8$} \\
\hline $\mathrm{VEF}_{1} / \mathrm{CVF}$ (\% do predito) & \multicolumn{2}{|c|}{$75,1(70,6 ; 84,7)$} \\
\hline \multicolumn{3}{|l|}{ Capacidade funcional (TC6) } \\
\hline Distância percorrida (m) & $453,93 \pm 73,85$ & $562,99 \pm 35,58^{*}$ \\
\hline
\end{tabular}

*diferença estatisticamente significante $(p<0,05)$ entre obtido e predito; PImáx: pressão inspiratória máxima; PEmáx: pressão expiratória máxima; CVF: capacidade vital forçada; $V_{E F}$ : volume expiratório forçado no primeiro segundo; TC6: teste de caminhada dos seis minutos

Tabela 5: Força muscular respiratória, função pulmonar e capacidade funcional dos pacientes divididos em dois grupos (estadiamento inicial e avançado) $(n=30)$

\begin{tabular}{|c|c|c|}
\hline & \multicolumn{2}{|c|}{$\begin{array}{l}\text { Estadiamento } \\
\text { Média } \pm \mathrm{DP} \text { ou mediana } \\
\left(1^{\circ} \text { e } 3^{\circ} \text { quartis }\right)\end{array}$} \\
\hline & Inicial $(n=15)$ & Avançado ( $n=15)$ \\
\hline \multicolumn{3}{|l|}{ Força muscular respiratória } \\
\hline Plmáx $\left(\mathrm{cmH}_{2} \mathrm{O}\right)$ & $76,0(60 ; 120)$ & $120(72 ; 120)$ \\
\hline PEmáx $\left(\mathrm{cmH}_{2} \mathrm{O}\right)$ & $83,06 \pm 26,92$ & $95,85 \pm 25,95$ \\
\hline \multicolumn{3}{|l|}{ Função pulmonar } \\
\hline CVF (L) & $2,89 \pm 0,61$ & $3,41 \pm 0,60^{*}$ \\
\hline $\operatorname{VEF}_{1}(\mathrm{~L})$ & $2,29 \pm 0,62$ & $2,57 \pm 0,51$ \\
\hline $\mathrm{VEF}_{1} / \mathrm{CVF}(\%)$ & $82,21(67,13 ; 89,14)$ & $74,08(71,52 ; 76,87)$ \\
\hline \multicolumn{3}{|c|}{ Capacidade funcional (TC6) } \\
\hline Distância percorrida (m) & $444,78 \pm 86,74$ & $486,66 \pm 44,9$ \\
\hline
\end{tabular}

*diferença estatisticamente significante $(p<0,05)$ entre os grupos; PImáx: pressão inspiratória máxima; PEmáx: pressão expiratória máxima; CVF: capacidade vital forçada; $V E F_{1}$ : volume expiratório forçado no primeiro segundo; TC6: teste de caminhada dos seis minutos

\section{DISCUSSÃO}

No presente estudo pelo menos $92 \%$ dos pacientes tiveram algum contato com o tabaco durante a vida; segundo o INCA ${ }^{1}$, o consumo de derivados do tabaco está na origem de $90 \%$ dos casos de câncer de pulmão. A cirurgia de ressecção pulmonar mais comum encontrada no estudo de Brandão et al. ${ }^{2}$ foi a lobectomia, assim como no presente estudo, sendo o tratamento cirúrgico sugerido em $53 \%$ dos pacientes da atual amostra.

Com referência à classificação do estadiamento dos tumores, esta se trata de ação essencial no diagnóstico do câncer, pois determina todo o planejamento terapêutico e indica o prognóstico do caso ${ }^{10}$. No presente estudo observa-se que a maioria dos pacientes estava no estadiamento IIIA (26,6\%). De acordo com Novaes et al. ${ }^{16}$, o estadiamento avançado reflete a sintomatologia tardia e a alta agressividade de alguns tipos histológicos. Vale salientar que todos os pacientes do presente estudo apresentavam critérios de operabilidade e foram submetidos ao procedimento cirúrgico.
Após o exame, todos os pacientes apresentaram redução da função pulmonar, da força muscular expiratória e da capacidade funcional no pré-operatório de ressecção em relação aos valores de normalidade estabelecidos na literatura para a população saudável. Quando esses pacientes foram divididos em grupos de estadiamento houve diferença estatística apenas para a CVF, em que os pacientes em estadiamento inicial apresentaram valores menores em relação aos de estadiamento avançado. Para as demais variáveis, os grupos se comportaram de maneira semelhante.

A realização de testes de função pulmonar e avaliação da capacidade funcional pré-operatória podem predizer a evolução e o risco de complicações pós-operatórias ${ }^{17,18}$. Em pacientes com $\mathrm{VEF}_{1}$ abaixo de $80 \%$ do predito, a função pulmonar pós-operatória pode ser calculada por estimativa da quantidade de funcionamento de tecido pulmonar que seria perdido com a ressecção cirúrgica, bem como a avaliação da capacidade funcional pode ser realizada pelo teste cardiopulmonar com medida direta do consumo máximo de oxigênio $\left(\mathrm{VO}_{2} \mathrm{máx}\right)$, pelo shuttle walking test ou pelo TC6 ${ }^{19}$.

Dentre os fatores de risco para complicações pulmonares após ressecção pulmonar estão: idade avançada (acima de 70 anos), tabagismo ativo, estado nutricional comprometido (IMC $<18,5)$, baixa reserva pulmonar $\left(\mathrm{VEF}_{1}<40 \%\right)$ e baixa capacidade de exercício $\left(\mathrm{VO}_{2} \text { máx }<15 \mathrm{~mL} / \mathrm{min}\right)^{18,20}$. O TC6 possui boa correlação com o $\mathrm{VO}_{2}$ máx obtido de forma direta pelo teste cardiopulmonar, considerado como padrão-ouro ${ }^{21}$.

Dessa forma, é possível realizar a triagem dos pacientes elegíveis para a cirurgia de ressecção pulmonar ${ }^{19,20}$, além de inserir o profissional fisioterapeuta na avaliação e no tratamento dos pacientes considerados com risco aumentado de complicações.

Diversos estudos demonstram que a cirurgia de ressecção pulmonar gera impacto na função pulmonar e na capacidade ao exercício ${ }^{5-7}$, podendo não ser recuperada em longo prazo, como demonstra o estudo de Win et al. ${ }^{5}$, no qual foi observada uma redução da função pulmonar e na distância percorrida no shuttle walk test no pós-operatório de ressecção pulmonar, permanecendo tal redução em até seis meses após a cirurgia, sendo ainda maior nas pneumectomias.

A caracterização da função pulmonar desses pacientes no pré-operatório é de grande importância para o fisioterapeuta traçar metas e direcionar o tratamento tanto no pré como no pós-operatório, no intuito de independência funcional e qualidade de vida após a cirurgia.

O tratamento fisioterápico desses pacientes no pós-operatório é importante e tem a finalidade de diminuir as complicações pulmonares e reabilitar funcionalmente em curto e longo prazo, demonstrando resultados satisfatórios, fazendo uso da inspirometria de incentivo ${ }^{22}$, da ventilação mecânica não invasiva ${ }^{23-25} \mathrm{e}$ de diversas modalidades de expansão pulmonar e remoção de secreções ${ }^{26,27}$, porém o presente estudo demonstra que tais pacientes possuem prejuízos na função pulmonar e na capacidade funcional já no pré-operatório, evidenciando a necessidade de intervenção 
profilática com o objetivo de preparar o paciente para a cirurgia de ressecção e, dessa forma, obter resultados ainda mais satisfatórios na reabilitação pós-operatória.

Nesse sentido, o estudo de Morano et al. ${ }^{28}$ demonstra aumento na capacidade funcional, na força muscular respiratória e na função pulmonar, e diminuição das complicações pós-operatórias com quatro semanas de tratamento em programa pré-operatório de reabilitação pulmonar em pacientes candidatos à cirurgia de ressecção pulmonar.

Conclui-se que os pacientes estudados com diagnóstico de neoplasia pulmonar, em qualquer estadiamento, no pré-operatório de ressecção pulmonar, possuem redução da força muscular expiratória, da função pulmonar e da capacidade funcional em relação aos valores previstos. Os pacientes com estadiamento inicial possuem redução da CVF.

Sugere-se maior atenção do fisioterapeuta aos pacientes em pré-operatório de ressecção pulmonar e implantação de programas de reabilitação pulmonar pré-operatória, para que o paciente esteja mais bem preparado para enfrentar a cirurgia e o pós-operatório. No entanto, há a necessidade de investigação e ampliação da literatura científica que evidenciem os benefícios de programas de reabilitação pré-operatória em ressecção pulmonar.

\section{REFERÊNCIAS}

1. Instituto Nacional do Câncer (INCA). Tipos de câncer: pulmão [Internet]. Disponivel em: http://www2.inca.gov.br/wps/wcm/connect/ tiposdecancer/site/home/pulmao/definicao. Acesso em: 31 ago. 2014.

2. Brandão DS, Haddad R, Marsico GA, Boasquevisque CHR. Aspectos clínico-patológicos do carcinoma bronquioloalveolar e sobrevida em pacientes no estágio clínico I. J Bras Pneumol. 2010;36(2):167-74

http://dx.doi.org/10.1590/S1806-37132010000200003

3. Secretan B, Straif K, Baan R, Grosse Y, El Ghissassi F, Bouvard V, et al. A review of human carcinogens - Part E: tobacco, areca nut, alcohol, coal smoke, and salted fish. Lancet Oncol 2009; 10(11):1033-4. http://dx.doi.org/10.1016/S1470-2045(09)70326-2

4. Jamnik S, Santoro IL, Borges EL, Uehara C, Silva VV. Estudo comparativo de fatores prognósticos em portadores de carcinoma não-pequenas células de pulmão: sobrevida superior a cinco anos e inferior a um ano. Rev Bras Cancerol. 2009;55(1):5-10.

5. Win T, Groves AM, Ritchie AJ, Wells FC, Cafferty F, Laroche CM. The effect of lung resection on pulmonary function and exercise capacity in lung cancer patients. Respir Care. 2007;52(6):720-6.

6. Lima LNT, Silva RA, Gross JL, Deheinzelin D, Negri EM. Avaliação da função pulmonar e da qualidade de vida em pacientes submetidos à ressecção pulmonar por neoplasia. J Bras Pneumol. 2009;35(6):521-8

http://dx.doi.org/10.1590/S1806-37132009000600005

7. Borges-Santos E, Genz ICH, Longo AF, Hayahsi D, Gonçalves $C G$, Bellinetti LM, et al. Comportamento da função pulmonar, força muscular respiratória e qualidade de vida em pacientes submetidos às toracotomias eletivas. Rev Col Bras Cir. 2012;39(1):4-9.

http://dx.doi.org/10.1590/S0100-69912012000100003

8. Stephan F, Boucheseiche S, Hollande J, Flahault A, Cheffi A, Bazelly $\mathrm{B}$, et al. Pulmonary complications following lung resection: a comprehensive analysis of incidence and possible risk factors. Chest. 2000;118(5):1263-70.

9. Bellinetti LM, Thomson JC. Avaliação muscular respiratória nas toracotomias e laparotomias superiores eletivas. J Bras Pneumol. 2006;32(2):99-105.

http://dx.doi.org/10.1590/S1806-37132006000200004

10. Brasil. Ministério da Saúde. Secretaria de Atenção à Saúde. Instituto Nacional de Câncer. TNM: classificação de tumores malignos. 6 ed. Rio de Janeiro: INCA; 2004 [Internet]. Disponível em: http://www. inca.gov.br/tratamento/tnm/tnm2.pdf. Acesso em: 10 dez. 2014.
11. Neder JA, Andreoni S, Lerario MC, Nery LE. Reference values for lung function tests. II. Maximal respiratory pressures and voluntary ventilation. Braz J Med Biol Res.1999;32(6):719-27. http://dx.doi.org/10.1590/S0100-879X1999000600007

12. Brusasco V, Crapo R, Viegi G. Series "ATS/ERS Task Force: standardisation of lung function testing". Eur Respir J. 2005;26(2):319-38.

http://dx.doi.org/10.1183/09031936.05.00034805

13. Pereira CAC, Sato T, Rodrigues SC. Novos valores de referência para espirometria forçada em brasileiros adultos de raça branca. J Bras Pneumol. 2007;33(4):397-406.

http://dx.doi.org/10.1590/\$1806-37132007000400008

14. ATS Committee on Proficiency Standards for Clinical Pulmonary Function Laboratories. ATS statement: guidelines for the SixMinute Walk Test. Am J Respir Crit Care Med. 2002;166(1):111-7. http://dx.doi.org/10.1164/ajrccm.166.1.at1102

15. Britto RR, Probst VS, Dornelas de Andrade AF, Samora GAR, Hernandes NA, Marinho PEM, et al. Reference equations for the six-minute walk distance based on a Brazilian multicenter study. Braz J Phys Ther. 2013;17(6):556-3

http://dx.doi.org/10.1590/S1413-35552012005000122

16. Novaes FT, Cataneo DC, Ruiz Junior RL, Defaveri J, Michelin OC Cataneo AJM. Lung cancer: histology, staging, treatment and survival. J Bras Pneumol. 2008;34(8):595-600.

17. Filardo FA, Faresin SM, Fernandes ALG. Validade de um índice prognóstico para ocorrência de complicações pulmonares no pós-operatório de cirurgia abdominal alta. Rev Assoc Med Bras. 2002;48(3):206-16

http://dx.doi.org/10.1590/S0104-42302002000300032

18. Degani-Costa LH, Faresin SM, Falcão LFR. Avaliação préoperatória do paciente pneumopata. Rev Bras Anestesiol. 2014:64(1):22-34

http://dx.doi.org/10.1016/j.bjan.2012.11.002

19. Colice GL, Shafazand S, Griffin JP, Keenan R, Bolliger CT; American College of Chest Physicians. Physiologic evaluation of the patient with lung cancer being considered for resectional surgery: ACCP evidenced-based clinical practice guidelines (2nd Edition). Chest. 2007:132(3 Suppl):161S-77. http://dx.doi.org/10.1378/chest.07-1359

20. Silva DR, Baglio PT, Gazzana MB, Barreto SSM. Avaliação pulmonar e prevenção das complicações respiratórias perioperatórias. Rev Bras Clin Med. 2009;7(2):114-23. 
21. Cataneo DC, Kobayasi S, Carvalho LR, Paccanaro RC, Cataneo AJM. Accuracy of six minute walk test, stair test and spirometry using maximal oxygen uptake as gold standard. Acta Cir Bras. 2010;25(2):194-200.

http://dx.doi.org/S0102-86502010000200013

22. Agostini $\mathrm{P}$, Calvert $\mathrm{R}$, Subramanian $\mathrm{H}$, Naidu B. Is incentive Spirometry effective following thoracic surgery? Interact Cardiovasc Thorac Surg. 2008;7(2):297-300 http://dx.doi.org/10.1510/icvts.2007.171025

23. Passos AIM, Baltieri L, Galhardo FDM, Roceto LS, Figueiredo LC, Toro IFC. Utilização da ventilação mecânica não invasiva no pós-operatório de ressecção pulmonar. Rev Saúde Pesquisa. 2013;6(3):399-407.

24. Lefebvre A, Lorut C, Alifano M, Dermine H, Roche N, Gauzit R, et al. Noninvasive ventilation for acute respiratory failure after lung resection: an observational study. Intensive Care Med. 2009;35(4):663-70. http://dx.doi.org/10.1007/s00134-008-1317-z
25. Perrin C, Jullien V, Vénissac N, Berthier F, Padovani B, Guillot F, et al. Prophylactic use of non-invasive ventilation in patients undergoing lung resectional surgery. Respir Med. 2007;101(7):1572-8. http://dx.doi.org/10.1016/j.rmed.2006.12.002

26. Lawrence VA, Cornell JE, Smetana GW. Strategies to reduce postoperative pulmonary complications after noncardiothoracic surgery: systematic review for the American College of Physicians. Ann Intern Med. 2006;144(8):596-608.

http://dx.doi.org/10.7326/0003-4819-144-8-200604180-00011

27. Canet J, Mazo V. Postoperative pulmonary complications. Minerva Anestesiol. 2010;76(2):138-43.

28. Morano MT, Araújo AS, Nascimento FB, Silva GF, Mesquita R, Pinto JS, et al. Preoperative pulmonary rehabilitation versus chest physical therapy in patients undergoing lung cancer resection: a pilot randomized controlled trial. Arch Phys Med Rehabil. 2013;94(1):53-8.

http://dx.doi.org/10.1016/j.apmr.2012.08.206 